\title{
Effects of combination treatment with transcranial magnetic stimulation and bone marrow mesenchymal stem cell transplantation or Raf inhibition on spinal cord injury in rats
}

\author{
SINING FENG, SHUAI WANG, SHI SUN, HAO SU and LIXIN ZHANG \\ Department of Rehabilitation, Shengjing Hospital of China Medical University, Shenyang, Liaoning 110004, P.R. China
}

Received September 2, 2020; Accepted January 7, 2021

DOI: $10.3892 / \mathrm{mmr} .2021 .11934$

\begin{abstract}
Spinal cord injury (SCI) remains a global challenge due to limited treatment strategies. Transcranial magnetic stimulation (TMS), bone marrow mesenchymal stem cell (BMSC) transplantation and downregulation of Raf/MEK/ERK signaling effectively improve SCI. The combination of BMSCs and TMS displays synergistic effects on vascular dementia. However, whether TMS displays a synergistic effect when combined with BMSC transplantation or Raf inhibitor (RafI) therapy for the treatment of SCI is not completely understood. The present study aimed to compare the therapeutic effect of monotherapy and combination therapy on SCI. In the present study, 8-week-old female Sprague Dawley rats were used to establish a model of SCI using the weight-drop method followed by treatment with monotherapy (TMS, BMSCs or RafI) or combination therapy (TMS+BMSCs or TMS+RafI). The effect of monotherapy and combination therapy on locomotor function, pathological alterations, neuronal apoptosis and expression of axonal regeneration-associated factors and Raf/MEK/ERK signaling-associated proteins in the spinal cord was analyzed by Basso, Beattie and Bresnahan (BBB) scoring, hematoxylin and eosin staining, TUNEL-neuronal nuclei (NeuN) staining and immunofluorescence or western blotting, respectively. The results demonstrated that compared with untreated SCI model rats, monotherapy significantly
\end{abstract}

Correspondence to: Dr Lixin Zhang, Department of Rehabilitation, Shengjing Hospital of China Medical University, 36 Sanhao Street, Shenyang, Liaoning 110004, P.R. China

E-mail: zhanglx@sj-hospital.org

Abbreviations: SCI, spinal cord injury; TMS, transcranial magnetic stimulation; BMSCs, bone marrow mesenchymal stem cells; NeuN, neuronal nuclei; GAP-43, growth-associated protein 43; NGF, nerve growth factor; BDNF, brain derived neurotrophic factor; GFAP, glial fibrillary acidic protein; TUNEL, TdT-mediated dUTP Nick-End Labeling; BBB scale, Basso, Beattie and Bresnahan locomotor rating scale

Key words: spinal cord injury, transcranial magnetic stimulation, bone marrow mesenchymal stem cell transplantation, Raf/MEK/ERK signaling, synergistic therapy enhanced locomotor functional recovery, as evidenced by higher BBB scores, and slightly alleviated histopathological lesions of the spinal cord in SCI model rats. Furthermore, monotherapy markedly suppressed neuronal apoptosis and promoted axonal regeneration, as well as inhibiting astroglial activation in SCI model rats. The aforementioned results were demonstrated by significantly decreased numbers of apoptotic neurons, markedly decreased expression levels of glial fibrillary acidic protein (GFAP), significantly increased numbers of $\mathrm{NeuN}^{+}$cells, markedly increased expression levels of growth-associated protein 43 (GAP-43) and significantly upregulated nerve growth factor (NGF) and brain derived neurotrophic factor (BDNF) expression levels in monotherapy groups (excluding the RafI monotherapy group) compared with untreated SCI model rats. In addition, monotherapy markedly suppressed activation of the Raf/MEK/ERK signaling pathway, as evidenced by significantly reduced p-Raf/Raf, p-MEK/MEK and p-ERK/ERK protein expression levels in monotherapy groups (excluding the BMSC monotherapy group) compared with untreated SCI model rats. Notably, combination therapy further alleviated SCI-induced spinal cord lesions and neuronal apoptosis, increased GAP-43, NGF and BDNF expression levels, downregulated GFAP expression levels and inhibited activation of the Raf/MEK/ERK signaling pathway in SCI model rats compared with the corresponding monotherapy groups. Therefore, it was hypothesized that compared with monotherapy, combination therapy displayed an improved therapeutic effect on SCI by further suppressing Raf/MEK/ERK signaling. The results of the present study provided an important basis for the clinical application of combination therapy.

\section{Introduction}

Despite efforts to promote spinal cord injury (SCI) repair (1), it is still regarded as an incurable condition with limited treatment strategies (2). SCI is a disabling injury condition that manifests as partial or complete loss of sensory and motor function below the level of injury $(3,4)$. Moreover, $>50 \%$ of SCIs result from trauma, including traffic accidents, sports injuries and gun shots, whereas other factors, such as inflammation, cancer and vascular diseases, are responsible for non-traumatic SCI (5). SCI results in a considerable burden on patients and treatment costs are high (6). The pathophysiology 
of SCI consists of both primary and secondary injury. Primary injury refers to the mechanical injury to the spinal cord, which includes central and peripheral nervous systems damage, microvasculature damage, axonal disruption, spinal cord swelling, spinal shock and hypotension (7). Secondary injury consists of excitotoxicity, free radical-induced lipid peroxidation, blood-brain barrier alterations, inflammation, and the death of neurons and glial cells (8). Therefore, the development of improved treatment strategies is important for patients with SCI.

Promoting axonal regeneration serves a pivotal role in SCI treatment (9). A previous study reported that astrocyte activation primarily mediates glial scar formation following SCI, which prevents axonal regeneration (10). Glial fibrillary acidic protein (GFAP) is a cytokine secreted by activated astrocytes, thus it is considered as a marker for astrocyte activation $(11,12)$. Growth-associated protein-43 (GAP-43, neuromodulin) participates in the regulation of axonal growth (13). Neurotrophins, including nerve growth factor (NGF) and brain-derived neurotrophic factor (BDNF), have been shown to promote axonal regrowth and regeneration of injured spinal cord (14). Therefore, GFAP, GAP-43, NGF and BNDF are often assessed to evaluate axonal regeneration.

Transcranial magnetic stimulation (TMS) is a medical technology based on bioelectromagnetics, which was first introduced by Barker et al (15). TMS refers to the stimulation of the central nervous system, particularly the brain, with a pulsed magnetic field (16). Due to its numerous advantages, including its non-invasive nature, convenience and lack of pain, interesting results have been observed for the use of TMS treatment for paralysis, Parkinson's disease, epilepsy and depression $(17,18)$. Increasing evidence demonstrates that TMS might serve as a potential neurophysiological technique for SCI diagnosis and treatment, and clinical trials have reported that high frequency repetitive TMS promotes motor rehabilitation and modulates brainstem reflexes in individuals with SCI $(19,20)$.

Embryonic, fetal neural and induced pluripotent stem cells may be used to repair SCI; however, the clinical application of stem cell therapies is limited by immunological rejection and ethical concerns (21-23). Bone marrow mesenchymal stem cells (BMSCs), which can be easily obtained from bone marrow, display the potential to differentiate into cartilage cells, osteoblasts, fat cells, myoblasts and nerve cells (24). BMSCs are widely used in cellular transplantation due to their easy acquisition, low immunogenicity and lack of ethical restraints (25). Additionally, a previous study demonstrated that BMSCs could differentiate into neuronal-like cells and promote SCI repair in mice (26). The Raf/MEK/ERK signaling pathway is a signaling cascade involved in various physiological processes, including cellular proliferation and differentiation (27). Raf/MEK/ERK signaling is also involved in neuronal apoptosis in the hippocampus following subarachnoid hemorrhage (28). Previous studies revealed that Raf/MEK/ERK signaling was upregulated in SCI model rats (29), and that the Raf inhibitor dabrafenib displays potential to facilitate SCI recovery (30). Therefore, the present study aimed to investigate the feasibility of allogenic BMSCs and Raf inhibition for SCI treatment. In the present study, a rat model was established to mimic SCI. Subsequently, rats were treated with BMSCs, Raf inhibitor (RafI), TMS, TMS+BMSC or TMS+RafI. The effects of monotherapy and combination therapy on functional recovery, pathological alterations, neuronal apoptosis and the expression levels of neuromodulin, neurotrophins, astrocyte markers and Raf/MEK/ERK signaling were assessed.

\section{Materials and methods}

Animal model establishment and treatment. Female Sprague Dawley rats (age, 8 weeks; weight, $220 \pm 20 \mathrm{~g}$ ) were purchased from Liaoning Changsheng Biotechnology Co., Ltd. Rats were housed in cages at $22 \pm 1^{\circ} \mathrm{C}$ with 12 -h light/dark cycles, $45-55 \%$ relative humidity, and ad libitum access to food and water. Following adaptive feeding for one week, 84 rats were randomly divided into the following 7 groups (12 rats per group): i) Sham; ii) SCI; iii) SCI+BMSCs; iv) SCI+RafI; v) SCI+TMS; vi) SCI+TMS+BMSCs; and vii) SCI+TMS+RafI. Rats were anesthetized by the intraperitoneal injection of $50 \mathrm{mg} / \mathrm{kg}$ Nembutal. Subsequently, the soft tissue around vertebral level T10 was removed to expose the spinous processes and laminae of T10 and part of T9/T11. Then, a 10-g impactor was used to induce spinal cord contusion injury (100 $\mathrm{g} \mathrm{x} \mathrm{cm} \mathrm{x} \mathrm{force)}$ by the weight-drop method (31). Following SCI induction, the incision was sutured layer by layer under sterilized conditions. Rats were intraperitoneally injected with penicillin (40,000 U/day/rat) for 3 days post-surgery, and the bladder of each rat was extruded for urination twice daily. In the sham group, incisions were directly sutured without SCI induction, and the rats did not undergo manual urination. Following SCI induction, rats received the following treatment: i) Rats in the sham group were injected at the same site with $5 \mu \mathrm{l}$ DMEM/F12 medium (Gibco; Thermo Fisher Scientific, Inc.) supplemented with $10 \%$ fetal calf serum (Hyclone; Cytiva) and $10 \mu \mathrm{l}$ normal saline on day 7 post-surgery, followed by $10 \mu 1$ normal saline daily from day $8-28$ post-surgery; ii) rats in the SCI group were injected into the SCI site with $5 \mu 1$ DMEM/F12 medium supplemented with $10 \%$ fetal calf serum and $10 \mu 1$ normal saline on day 7 post-surgery, followed by $10 \mu 1$ normal saline daily from day $8-28$ post-surgery; iii) rats in the SCI+BMSCs group were injected into the SCI with $5 \mu 1$ BMSC suspension $\left(1 \times 10^{5}\right.$ cells $\left./ \mu 1\right)$ and $10 \mu 1$ normal saline on day 7 post-surgery, followed by $10 \mu \mathrm{l}$ normal saline daily from day 8-28 post-surgery; iv) rats in the SCI+RafI group were injected into the SCI site with $5 \mu \mathrm{l}$ DMEM/F12 medium supplemented with $10 \%$ fetal calf serum and $10 \mu 1$ PLX4720 ( $1 \mu \mathrm{g} / \mu \mathrm{l}$; Aladdin) on day 7 post-surgery, followed by $10 \mu \mathrm{l}$ PLX4720 daily from day 8-28 post-surgery; v) following stimulation with $10 \mathrm{~Hz}$ TMS at $24 \mathrm{~h}$ post-surgery, and then once a day, 5 days a week, for 4 weeks, rats in the SCI+TMS group were injected into the SCI site with $5 \mu \mathrm{l}$ DMEM/F12 medium supplemented with $10 \%$ fetal calf serum and $10 \mu \mathrm{l}$ normal saline on day 7 post-surgery, followed by $10 \mu$ normal saline daily from day 8-28 post-surgery; vi) rats in the SCI+TMS+BMSCs group received the same TMS treatment as the SCI+TMS group, and were injected into the SCI site with $5 \mu \mathrm{l}$ BMSC suspension $\left(1 \times 10^{5}\right.$ cells $\left./ \mu 1\right)$ and $10 \mu \mathrm{l}$ normal saline on day 7 post-surgery, followed by $10 \mu \mathrm{l}$ normal saline daily from day 8-28 post-surgery; vii) rats in the SCI+TMS+RafI group received the same TMS treatment as the SCI+TMS group, and 
were injected into the SCI site with $5 \mu \mathrm{l}$ DMEM/F12 medium supplemented with $10 \%$ fetal calf serum and $10 \mu \mathrm{l}$ PLX4720 $(1 \mu \mathrm{g} / \mu 1)$ on day 7 post-surgery, followed by $10 \mu 1$ PLX4720 daily from day $8-28$ post-surgery.

Rats were evaluated using the Basso, Beattie and Bresnahan locomotor rating (BBB) scale before surgery and at day 1,14 and 28 post-surgery $(32,33)$. A total of six live rats per group were used for BBB scoring and subsequent experiments. Subsequently, rats were euthanized by the intraperitoneal injection of $200 \mathrm{mg} / \mathrm{kg} \mathrm{Nembutal}$. A total of six rats were used to assess hematoxylin and eosin (H\&E) staining, TUNEL and neuronal nuclei (NeuN) double staining as well as immunofluorescence staining; another six rats were used to perform western blotting. Spinal cord tissues were fixed with $4 \%$ paraformaldehyde at room temperature for $48 \mathrm{~h}$ or stored at $-70^{\circ} \mathrm{C}$. All animal experiments were performed according to Guide for the Care and Use of Laboratory Animals 8th edition (34), and approved by the ethics committee of Shengjing Hospital of China Medical University (approval no. 2020PS697K).

Isolation and identification of BMSCs. Femurs and tibias were collected from each rat and the soft tissues surrounding the femurs and tibias were removed. The marrow cavity was repeatedly syringed with DMEM/F12 culture medium to harvest bone marrow cells. Bone marrows cells were resuspended and lysed using red cell lysis buffer (Beijing Solarbio Science \& Technology Co., Ltd.). Following washing twice with PBS, cells were resuspended in DMEM/F12 culture medium supplemented with $10 \%$ fetal calf serum. Then, cells $\left(3 \times 10^{5} /\right.$ well $)$ were seeded into a 6 -well plate. For identification, BMSCs were collected at passage 3 and incubated with anti-CD11b (cat. no. 12-0110-80), anti-CD29 (cat. no. 11-0291-80), anti-CD90 (cat. no. 11-0900-81) and anti-CD45 (cat. no. 11-0461-80) monoclonal antibodies (all eBioscience; Thermo Fisher Scientific, Inc.) at $37^{\circ} \mathrm{C}$ for $30 \mathrm{~min}$. BMSCs were identified by flow cytometric detection (35) using an Accuri C6 flow cytometer (BD Biosciences) and Accuri C6 software (version 1.0.264.21; BD Biosciences).

$H \& E$ staining. Rat spinal cord tissues were embedded in paraffin and cut into 5- $\mu \mathrm{m}$ thick sections. Tissue sections were stained using a H\&E staining kit (Wanleibio Co., Ltd.) according to the manufacturer's instructions. Stained sections were visualized using a fluorescence microscope (Olympus Corporation; magnification, $\mathrm{x} 400$ ).

TUNEL and NeuN double staining. Paraffin-embedded spinal cord tissues were cut into 5- $\mu \mathrm{m}$ thick sections, de-paraffinized by heating to $60^{\circ} \mathrm{C}$ for $2 \mathrm{~h}$ followed by xylene immersion, re-hydrated in a descending ethanol series $(95,85$, and $75 \%)$ and washed with PBS. Following heating in a microwave (low power) with citrate buffer containing 0.0018 citric acid and $0.0082 \mathrm{~mol} / 1$ sodium citrate for $10 \mathrm{~min}$ for antigen revival, tissue sections were stained using the In Situ Cell Death Detection Kit (Roche Diagnostics) according to the manufacturer's protocol. After washing with PBS, the tissue sections were blocked with 100\% goat serum (Beijing Solarbio Science $\&$ Technology Co., Ltd.) for $30 \mathrm{~min}$ at room temperature and then incubated with an anti-NeuN mouse monoclonal primary antibody (cat. no. ab104224; 1:400; Abcam) overnight at $4^{\circ} \mathrm{C}$.
After washing with PBS, tissue sections were incubated with a Cy3-labelled goat anti-mouse $\operatorname{IgG}(\mathrm{H}+\mathrm{L})$ secondary antibody (cat. no. A0521; 1:200; Beyotime Institute of Biotechnology) for $1 \mathrm{~h}$ at room temperature. Tissue sections were then stained with DAPI for $5 \mathrm{~min}$ at room temperature (Biosharp Life Sciences). Following sealing with anti-fluorescence quenching reagent (Beijing Solarbio Science \& Technology Co., Ltd.), stained tissue sections were observed using a fluorescence microscope (Olympus Corporation; magnification, x400) and three random fields of view were observed for each section. The quantification procedure was conducted by Image-pro plus software (version 6.0; Media Cybernetics, Inc.).

Immunofluorescence staining. Paraffin-embedded spinal cord tissues were cut into 5- $\mu \mathrm{m}$ thick sections, de-paraffinized by heating to $60^{\circ} \mathrm{C}$ for $2 \mathrm{~h}$ followed by xylene immersion, re-hydrated in a descending ethanol series $(95,85$ and $75 \%$ ), washed with PBS, heated in a microwave (low power) with citrate buffer for $10 \mathrm{~min}$ for antigen revival. Following washing with PBS, tissue sections were blocked with $100 \%$ goat serum for $15 \mathrm{~min}$ at room temperature. Subsequently, tissue sections were incubated with anti-GFAP rabbit polyclonal (cat. no. WL0836; 1:100; Wanleibio Co. Ltd.) or anti-GAP-43 rabbit monoclonal (cat. no. ab75810; 1:200; Abcam) primary antibodies overnight at $4^{\circ} \mathrm{C}$. Following primary antibody incubation, tissue sections were incubated with Cy3-labelled goat anti-rabbit $\operatorname{IgG}(\mathrm{H}+\mathrm{L})$ secondary antibody (cat. no. A0516; 1:300; Beyotime Institute of Biotechnology) for $1 \mathrm{~h}$ at room temperature. After staining with DAPI for $5 \mathrm{~min}$ at room temperature, tissue sections were sealed with anti-fluorescence quenching reagent and stained sections were observed using a fluorescence microscope (Olympus Corporation; magnification, $\mathrm{x} 400)$.

Western blotting. Total protein was extracted from rat spinal cord tissues using the Whole Cell Lysis Assay Kit (Wanleibio Co., Ltd.) and quantified using the BCA Protein Assay Kit (Wanleibio Co., Ltd.). Proteins (40 $\mu \mathrm{g}$ per well) were separated via $8-12 \%$ SDS-PAGE and transferred onto PVDF membranes (EMD Millipore). After blocking with 5\% skimmed milk in TBST $(0.15 \%$ Tween- 20$)$ at room temperature for $1 \mathrm{~h}$, the membranes were incubated overnight at $4^{\circ} \mathrm{C}$ with the following primary antibodies: Rabbit polyclonal anti-NGF (cat. no. A13922; 1:1,000; ABclonal Biotech Co., Ltd.), rabbit monoclonal anti-BDNF (cat. no. BM4113; 1:200; Wuhan Boster Biological Technology, Ltd.), rabbit monoclonal anti-C-Raf (cat. no. BM4108; 1:1,000; Wuhan Boster Biological Technology, Ltd.), rabbit monoclonal anti-phosphorylated (p)-C-Raf (phospho ser259; cat. no. Bsm-52194R; 1:1,000; BIOSS), rabbit polyclonal anti-MEK1/2 (cat. no. WL02258; 1:500; Wanleibio Co. Ltd.), rabbit monoclonal anti-p-MEK1/2 (phosphor ser221; cat. no. 2338; 1:2,000; Cell Signaling Technology, Inc.), rabbit monoclonal anti-ERK1/2 (cat. no. BM4326; 1:200; Wuhan Boster Biological Technology, Ltd.), rabbit polyclonal anti-p-ERK1/2 (phospho thr202/tyr204; cat. no. WLP1512; 1:500; Wanleibio Co. Ltd.) and rabbit polyclonal anti- $\beta$-actin (cat. no. WL01845; 1:1,000; Wanleibio Co. Ltd.). Following washing with TBST, the membranes were incubated with HRP-conjugated goat anti-rabbit IgG secondary antibody (1:5,000; cat. no. WLA023; Wanleibio Co. Ltd.) for 

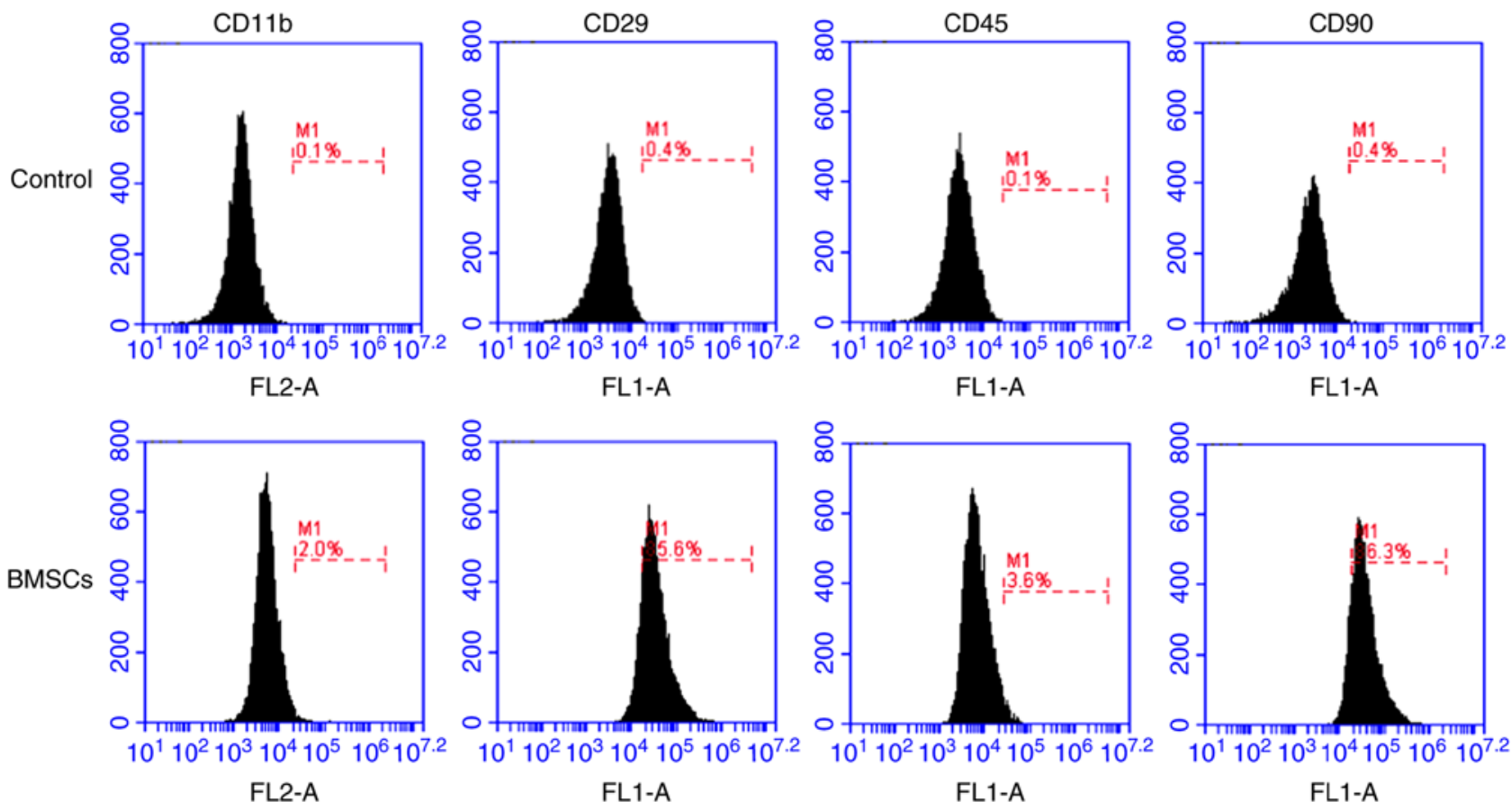

Figure 1. Identification of BMSCs. BMSCs were isolated from the rat bone marrow cavity and identified via flow cytometry. BMSCs were CD11b ${ }^{-}$CD45 $\mathrm{CD} 29^{+}$and $\mathrm{CD} 90^{+}$. BMSCs, bone marrow mesenchymal stem cells.

$45 \mathrm{~min}$ at $37^{\circ} \mathrm{C}$. Proteins were visualized using ECL luminescent reagent (Wanleibio Co. Ltd.). Protein expression levels were semi-quantified using Gel-Pro-Analyzer (version 4.0; Media Cybernetics, Inc.) with $\beta$-actin as the loading control.

Statistical analysis. Statistical analyses were performed using GraphPad Prism 7 software (GraphPad Software, Inc.). Data are presented as the mean $\pm \mathrm{SD}(n \geq 6)$. BBB scores among the 7 groups were compared using the Kruskal-Wallis test followed by Dunn's post hoc test. Comparisons among multiple groups were analyzed using one-way ANOVA followed by Tukey's post hoc test. $\mathrm{P}<0.05$ was considered to indicate a statistically significant difference.

\section{Results}

Successful isolation of BMSCs. BMSCs were isolated from rat bone marrow and identified via flow cytometry at passage 3 . The flow cytometry results demonstrated that the isolated cells were $\mathrm{CD}_{11 \mathrm{~b}}^{-}, \mathrm{CD} 45^{-}, \mathrm{CD} 29^{+}$and $\mathrm{CD}^{\circ} 0^{+}$(Fig. 1), indicating that the isolated cells were BMSCs.

Combination treatment of TMS+BMSCs or TMS+RafI further alleviates locomotor function, pathological alterations and neuronal apoptosis in SCI model rats. To investigate the effect of TMS+BMSCs and TMS+RafI combination therapies on SCI model rats, BBB scoring was employed to evaluate functional recovery (Fig. 2A). Following monotherapy or combination therapy, the BBB scores of SCI model rats at day 28 post-surgery were significantly increased (SCI+BMSCs, 14.00; SCI+RafI, 14.33; SCI+TMS, 13.17; SCI+TMS+BMSCs, 15.33; and SCI+TMS+RafI, 16.17) compared with untreated SCI model rats (7.83), which suggested that all therapies were beneficial for the recovery of locomotor function following
SCI. In addition, morphological alterations in the spinal cord were detected by performing H\&E staining. The spinal cord tissues of the SCI group displayed a little congestion and obvious structural disorders at the site of injury, whereas monotherapy slightly alleviated the intensity of spinal cord lesions (Fig. 2B). TMS+BMSCs and TMS+RafI combination therapies notably relieved SCI-induced structural damage (Fig. 2B). Neuronal apoptosis was determined by conducting TUNEL-NeuN double staining. The SCI group displayed a significantly increased number of apoptotic cells $(28.94 \%)$ and fewer $\mathrm{NeuN}^{+}$cells $(6.85 \%)$ compared with the sham group (TUNEL ${ }^{+}$cells, 1.19\%; $\mathrm{NeuN}^{+}$cells, 26.58\%), indicating that SCI enhanced neuronal apoptosis (Fig. 3A-C). However, compared with the SCI group, all therapies significantly increased the number of $\mathrm{NeuN}^{+}$cells (SCI+BMSCs, 15.09\%; SCI+RafI, 14.51\%; SCI+TMS, $13.81 \%$; SCI+TMS+BMSCs, $23.26 \%$; SCI+TMS+RafI, 23.10\%) and decreased the number of $\mathrm{TUNEL}^{+}$cells (SCI+BMSCs, $11.66 \%$; SCI+RafI, $15.97 \%$; SCI+TMS, $11.55 \%$; SCI+TMS+BMSCs, $6.75 \%$; SCI+TMS+RafI, $1.88 \%$ ), which was significantly enhanced in the SCI+TMS+BMSCs and SCI+TMS+RafI groups compared with the corresponding monotherapy groups (Fig. 3A-C). Collectively, the results suggested that the combination therapies were more effective at alleviating SCI-induced pathological alterations and neuronal apoptosis in SCI model rats compared with monotherapy.

Combination treatment of TMS+BMSCs or TMS+RafI further increases the expression of neuromodulin and neurotrophins, and decreases the expression of astrocyte markers in SCI model rats. GAP-43 and GFAP expression levels were detected by performing an immunofluorescence assay. GAP-43 expression levels were notably lower in the SCI group compared with the sham group (Fig. 4A). After 


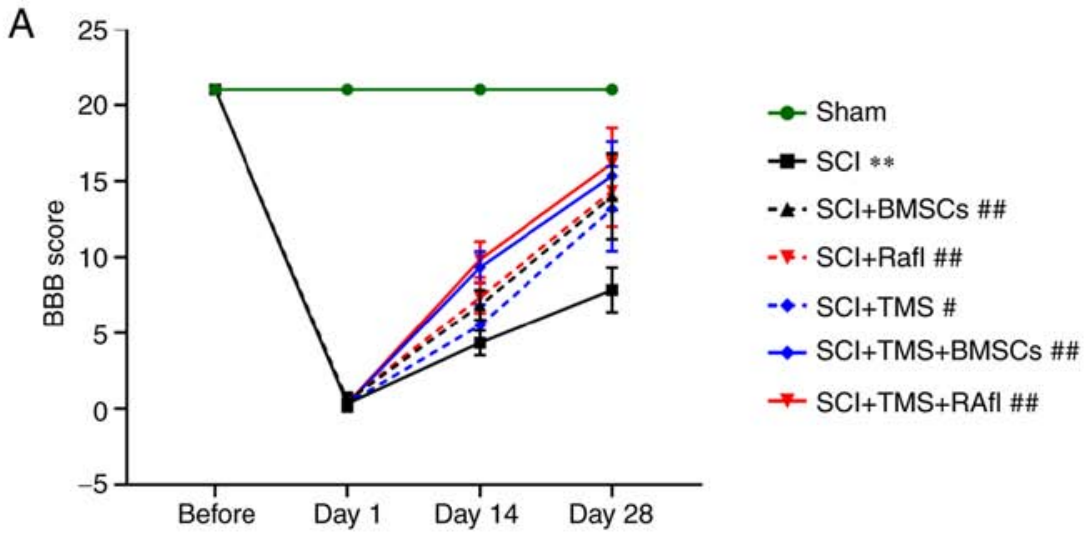

B
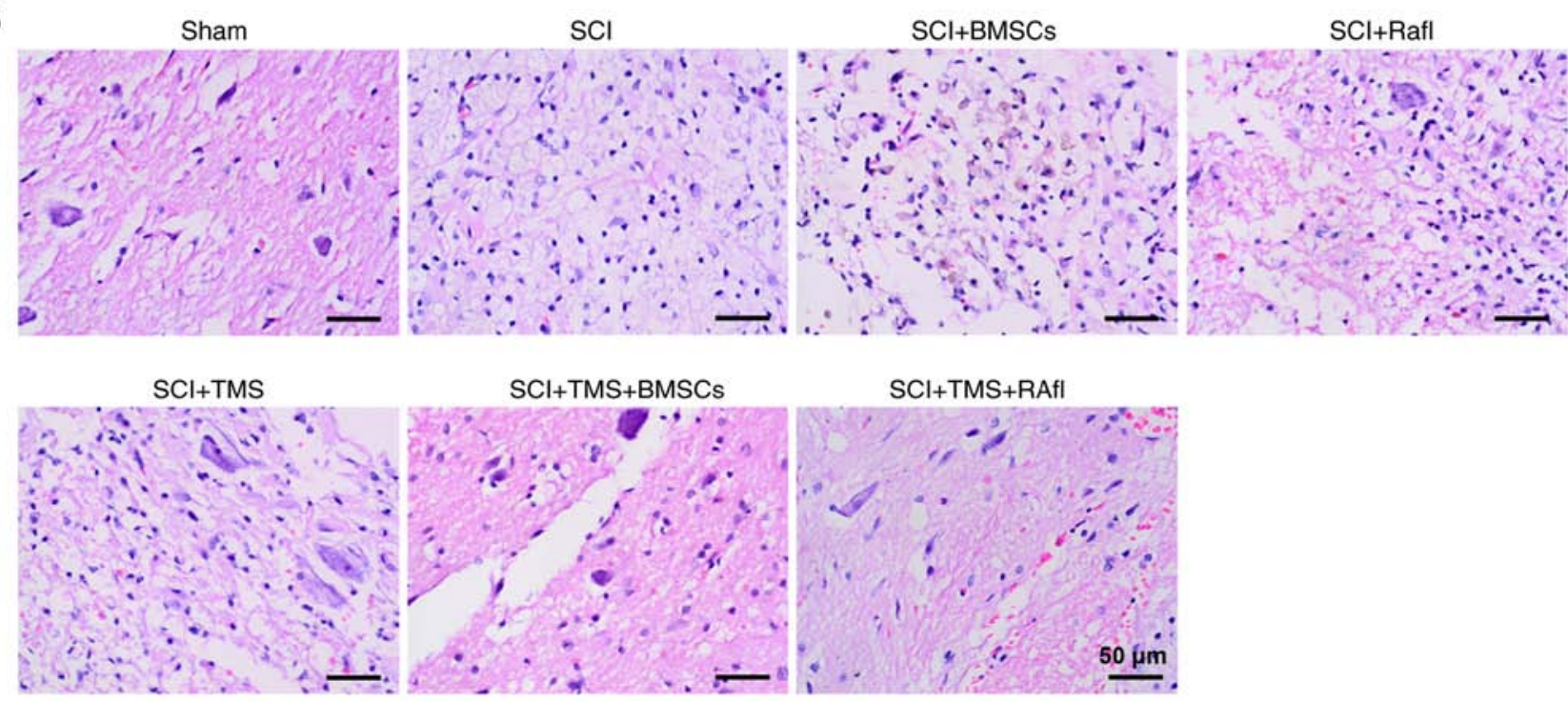

Figure 2. Effects of combination therapy on locomotor functional recovery and pathological alterations in SCI model rats. (A) BBB scoring was used to evaluate the recovery of locomotor function. (B) Hematoxylin and eosin staining was performed to evaluate spinal injury (scale bar, $50 \mu \mathrm{m}$ ). Green arrow, neurons; red arrow, congestion; yellow asterisk, vacuolation. ${ }^{* * *} \mathrm{P}<0.01$ vs. sham (day 28 ); ${ }^{*} \mathrm{P}<0.05$ and ${ }^{\# \#} \mathrm{P}<0.01$ vs. SCI (day 28 ). SCI, spinal cord injury; BBB, Basso, Beattie and Bresnahan locomotor rating; TMS, transcranial magnetic stimulation; BMSCs, bone marrow mesenchymal stem cells; RafI, Raf inhibitor.

monotherapy or combination therapy, GAP-43 expression was markedly increased in SCI model rats, which was more notable in the SCI+TMS+BMSCs and SCI+TMS+RafI groups (Fig. 4A). By contrast, the expression of GFAP was obviously upregulated in the SCI group compared with the sham group, which was decreased following monotherapy or combination therapy, where the reduction was more remarkable in the RafI, SCI+TMS+BMSCs and SCI+TMS+RafI groups (Fig. 4B). Subsequently, the relative expression levels of NGF and BDNF were detected via western blotting (Fig. 5A and B). With the exception of RafI monotherapy, all other monotherapies and combination therapies significantly increased the relative expression levels of NGF (SCI+BMSCs, 3.78; SCI+TMS, 4.4; SCI+TMS+BMSCs, 6.02; SCI+TMS+RafI, 7.52) and BDNF (SCI+BMSCs, 2.00; SCI+TMS, 2.02; SCI+TMS+BMSCs, 2.86; SCI+TMS+RafI, 3.05) in SCI model rats compared with untreated SCI model rats (NGF, 2.10; and BDNF, 1.12). Compared with the SCI group, RafI monotherapy increased the relative expression of NGF (3.26) and significantly upregulated BDNF (1.99) expression levels (Fig. 5A and B). Moreover, the increases in NGF and BDNF expression levels were significantly enhanced in the combination therapy groups compared with the corresponding monotherapy groups (Fig. 5A and B).

Combination treatment of TMS+BMSCs or TMS+RafI further inhibits Raf/MEK/ERK signaling in SCI model rats. The relative expression levels of Raf, p-Raf, MEK, p-MEK, ERK and p-ERK were detected (Fig. 6). Compared with the SCI group (p-Raf/Raf, 6.11; p-ERK/ERK, 4.51), all therapies significantly reduced the relative expression levels of p-Raf/Raf (SCI+BMSCs, 4.69; SCI+RafI, 3.56; SCI+TMS, 4.57; SCI+TMS+BMSCs, 3.22; SCI+TMS+RafI, 2.07) and p-ERK/ERK (SCI+BMSCs, 3.08; SCI+RafI, 2.35; SCI+TMS, 3.12; SCI+TMS+BMSCs, 1.93; SCI+TMS+RafI, 1.36). With the exception of BMSC monotherapy (4.14), all other therapies significantly reduced the relative expression levels of p-MEK/MEK (SCI+RafI, 3.09; SCI+TMS, 2.86; SCI+TMS+BMSCs, 1.66; SCI+TMS+RafI, 1.39) compared with the SCI group (4.58). BMSC monotherapy downregulated the relative expression levels of $\mathrm{p}-\mathrm{MEK} / \mathrm{MEK}$ compared with the SCI group. The downregulation of p-Raf/Raf,p-MEK/MEK and p-ERK/ERK expression levels was significantly enhanced in the combination treatment groups compared with the 

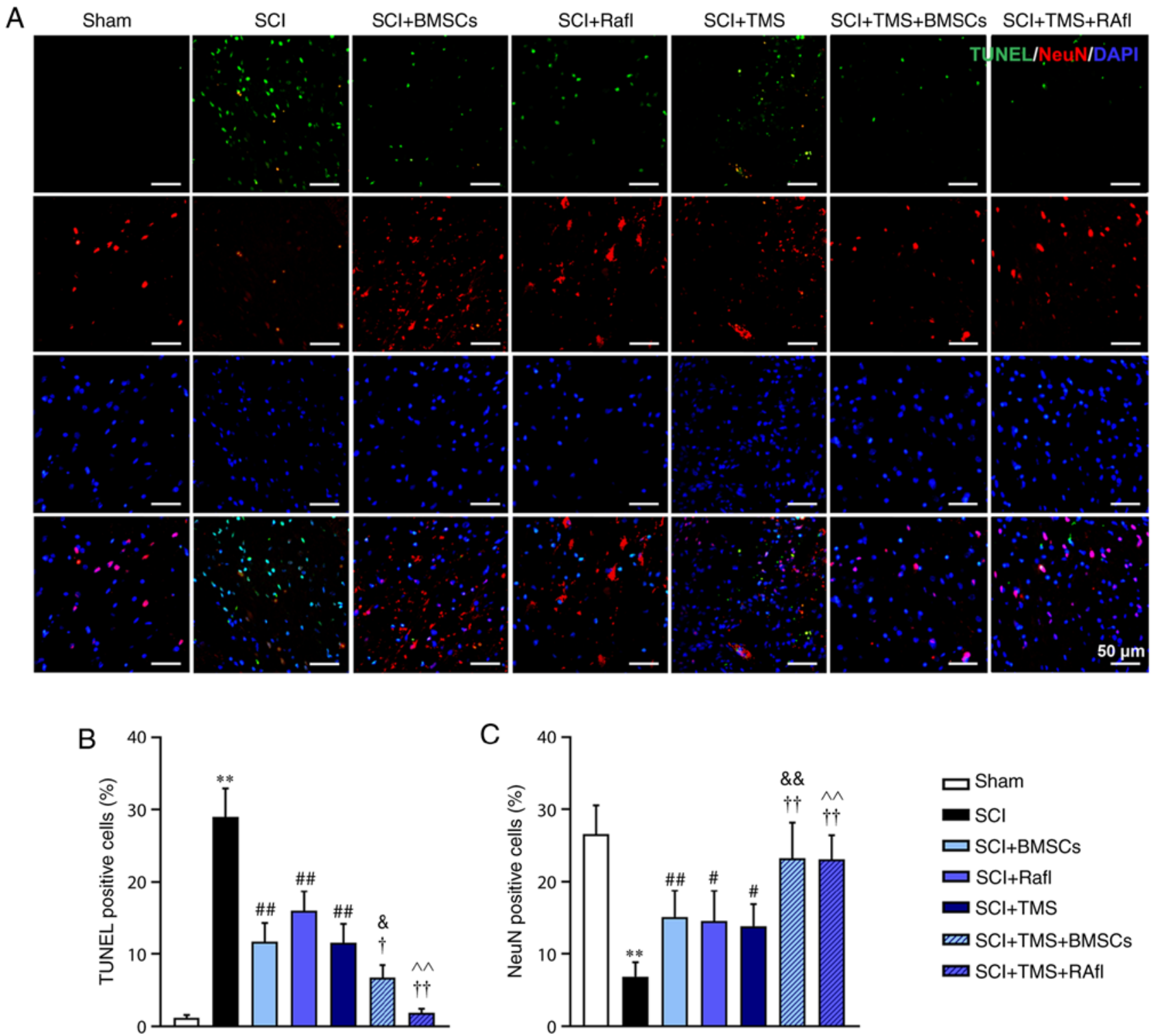

Figure 3. Effects of combination therapy on neuronal apoptosis in SCI model rats. (A) TUNEL and NeuN double staining were performed to evaluate neuronal apoptosis (scale bar, $50 \mu \mathrm{m}$ ). Quantification of (B) TUNEL ${ }^{+}$and $(\mathrm{C}) \mathrm{NeuN}^{+}$cells. ${ }^{* *} \mathrm{P}<0.01$ vs. sham; ${ }^{*} \mathrm{P}<0.05$ and ${ }^{\# \#} \mathrm{P}<0.01 \mathrm{vs}$. SCI; ${ }^{\dagger} \mathrm{P}<0.05$ and ${ }^{\dagger} \mathrm{P}<0.01$ vs. SCI+TMS; ${ }^{\circledR} \mathrm{P}<0.05$ and ${ }^{\& \&} \mathrm{P}<0.01$ vs. SCI+BMSCs; ${ }^{\wedge} \mathrm{P}<0.01$ vs. SCI+RafI. SCI, spinal cord injury; NeuN, neuronal nuclei; TMS, transcranial magnetic stimulation; BMSCs, bone marrow mesenchymal stem cells; RafI, Raf inhibitor.

corresponding monotherapy groups. No notable alterations in the relative expression levels of Raf, MEK and ERK were observed among the treatment groups.

\section{Discussion}

SCI commonly results from accidents and acts of violence, and patients with SCI experience a series of complications, including cerebrovascular damage, cervical spine injury and brain injury $(36,37)$. At present, there is no satisfactory treatment for SCI (2). Since its discovery in 1985, TMS has been widely used to treat a diverse range of neurodegenerative disorders (15). Previous studies have suggested that TMS might serve as a promising therapeutic strategy for $\operatorname{SCI}(38,39)$. BMSCs, which do not result in immunological rejection, are appropriate for the treatment of SCI (40). Emerging evidence has also demonstrated that BMSCs display the potential to regenerate neural function (41), and a previous study reported that BMSCs and TMS exerted synergistic effects in vascular dementia model rats (42). Recovery of the memory and learning ability of rats with vascular dementia was more significant following the combined application of TMS and BMSCs compared with either therapy alone, indicating that the combination of TMS and BMSCs may be more effective for the treatment of neurological diseases (42). However, whether the combined use of TMS and BMSCs is more effective compared with either therapy alone in treating SCI is not completely understood. Raf inhibition has also been reported as a potential therapeutic strategy for SCI (30), but whether the combination of TMS and Raf inhibition displays synergistic effects on SCI recovery requires further investigation. Therefore, the present study aimed to compare the effects of TMS, BMSC transplantation, RafI, TMS+BMSCs and TMS+RafI treatment on SCI in vivo. 

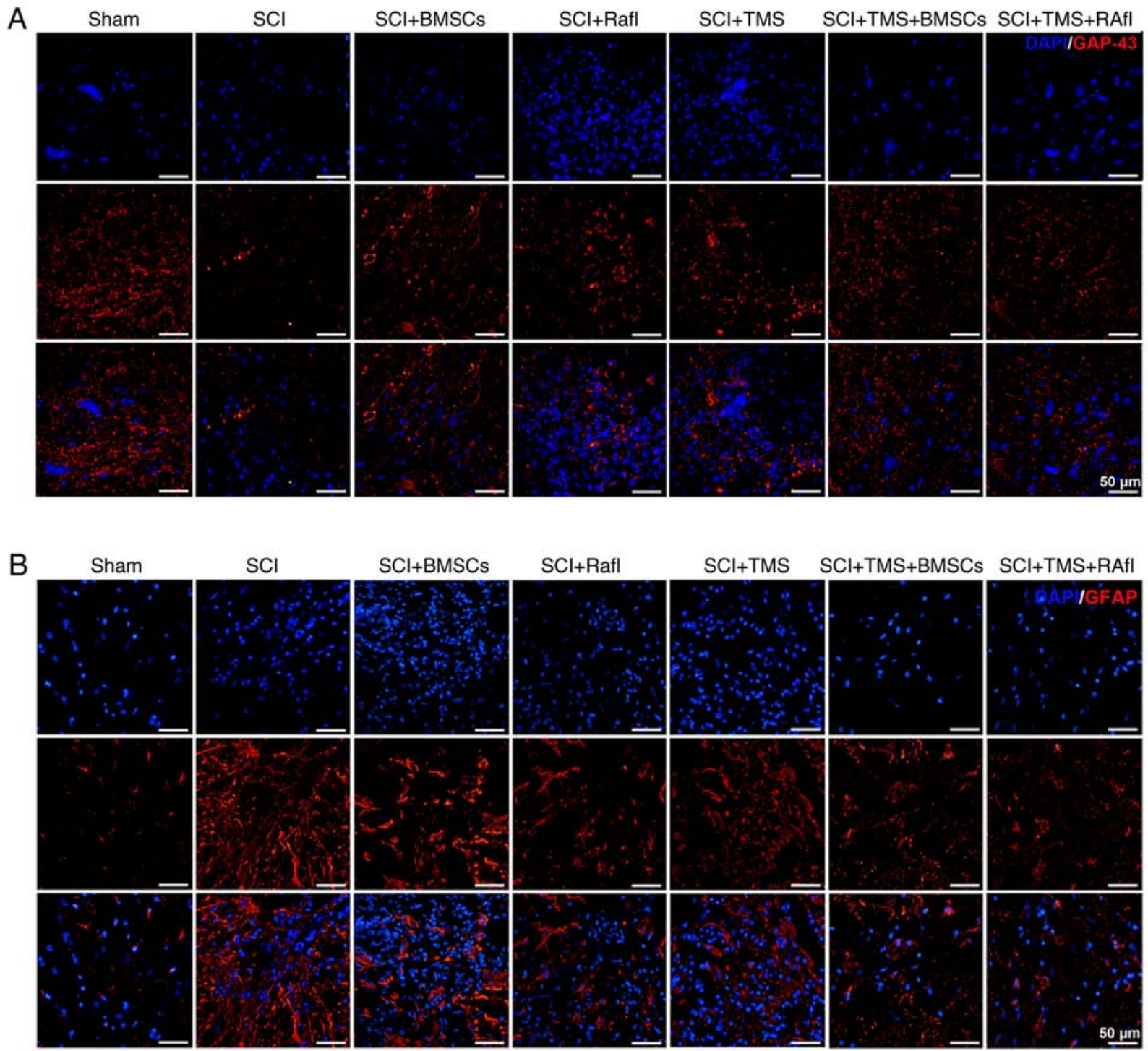

Figure 4. Effects of combination therapy on GAP-43 and GFAP expression in SCI model rats. (A) GAP-43 and (B) GFAP expression was evaluated via immunofluorescence staining (scale bar, $50 \mu \mathrm{m}$ ). GAP-43, growth-associated protein 43; GFAP, glial fibrillary acidic protein; SCI, spinal cord injury; TMS, transcranial magnetic stimulation; BMSCs, bone marrow mesenchymal stem cells; RafI, Raf inhibitor.

SCI can result in long-term motor deficits, thus the recovery of motor dysfunction is vital for patients with SCI (43). The BBB scale is commonly used to evaluate motor function deficit and recovery from various injuries (44). Furthermore, TMS was reported to facilitate motor recovery in rats with T10-11 vertebrae injuries, as evidenced by a higher BBB score in the TMS-treated group (45). Moreover, BMSC transplantation treatment displayed a significant effect on motor function recovery (46), which was consistent with the results of the present study. In the present study, the BBB scores of SCI model rats receiving TMS, BMSC or RafI treatment alone were significantly higher compared with untreated SCI model rats. Although the combined application of TMS+BMSCs or TMS+RafI did not further improve motor recovery in SCI model rats, the two combined treatments displayed no negative effects on motor recovery. In the present study, two key limitations were that the time interval for BBB scoring was potentially too long and there was a lack of additional time points. Moreover, histopathological lesions of the spinal cord were also attenuated by monotherapy and combination therapy to varying degrees.

Apoptosis, programmed cell death, is the process of cellular self-destruction (47). Apoptosis is implicated in secondary spinal cord injury, further influencing neural damage and functional deficits (48). In the present study, all therapies inhibited SCI-induced neuronal apoptosis, as evidenced by the decreased number of $\mathrm{TUNEL}^{+}$cells and the increased number of $\mathrm{NeuN}^{+}$cells in the treatment groups compared with untreated SCI model rats. Notably, the combined application of TMS+BMSCs or TMS+RafI significantly decreased the number of $\mathrm{TUNEL}^{+}$cells and significantly increased the number of $\mathrm{NeuN}^{+}$cells compared with the corresponding monotherapy groups, indicating that combination treatment displayed synergistic effects on the attenuation of neuronal apoptosis at the site of SCI. Apoptosis is one of the key determining factors of the extent of neuronal loss post-SCI (49), thus blocking neuronal apoptosis may improve functional recovery (50-52). Therefore, the attenuated functional deficits 

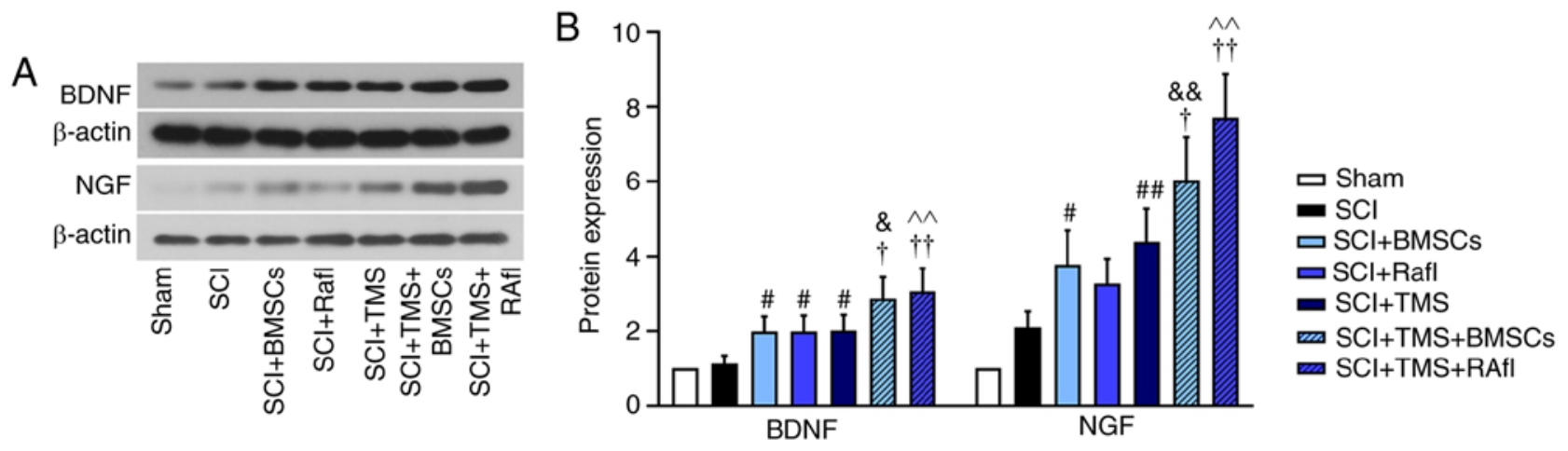

Figure 5. Effects of combination therapy on NGF and BDNF expression in SCI model rats. NGF and BDNF protein expression levels were (A) determined via western blotting and (B) semi-quantified. ${ }^{~} \mathrm{P}<0.05$ vs. and ${ }^{\# \#} \mathrm{P}<0.01$ vs. $\mathrm{SCI} ;{ }^{\dagger} \mathrm{P}<0.05$ and ${ }^{\dagger+} \mathrm{P}<0.01$ vs. SCI+TMS; ${ }^{8} \mathrm{P}<0.05$ and ${ }^{\text {\& }} \mathrm{P}<0.01$ vs. SCI+BMSCs; ${ }^{\wedge} \mathrm{P}<0.01$ vs. SCI+RafI. NGF, nerve growth factor; BDNF, brain derived neurotrophic factor; SCI, spinal cord injury; TMS, transcranial magnetic stimulation; BMSCs, bone marrow mesenchymal stem cells; RafI, Raf inhibitor.
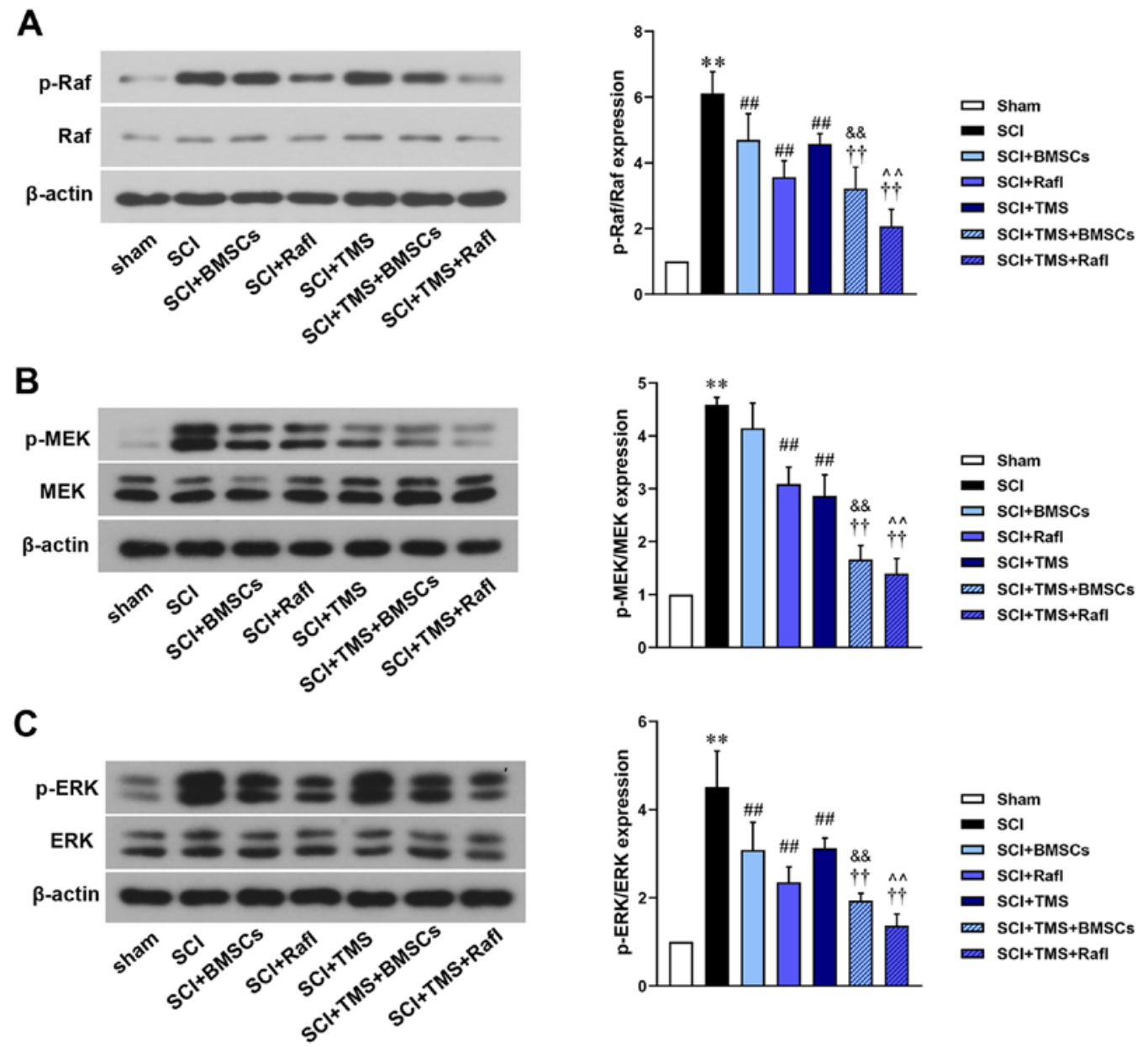

Figure 6. Effects of combination therapy on Raf/MEK/ERK signaling in SCI model rats. Protein expression levels of (A) p-Raf/Raf, (B) p-MEK/MEK and (C) $\mathrm{p}$-ERK/ERK were determined via western blotting. ${ }^{* *} \mathrm{P}<0.01$ vs. sham; ${ }^{\# \#} \mathrm{P}<0.01 \mathrm{vs}$. SCI; ${ }^{\dagger} \mathrm{P}<0.01 \mathrm{vs}$. SCI+TMS; ${ }^{* \&} \mathrm{P}<0.01$ vs. SCI+BMSCs; ${ }^{\wedge} \mathrm{P}<0.01 \mathrm{vs}$. SCI+RafI. SCI, spinal cord injury; p, phosphorylated; TMS, transcranial magnetic stimulation; BMSCs, bone marrow mesenchymal stem cells; RafI, Raf inhibitor.

induced by the therapies investigated in the present study may be partly attributed to inhibition of neuronal apoptosis.

GAP-43 is primarily expressed in developing and regenerating neurons, which is associated with neuronal growth (53). The astrocyte marker GFAP is involved in astroglial development and differentiation (54). In the present study, compared with untreated SCI model rats, all therapies markedly upregulated the expression of GAP-43 and notably downregulated the expression of GFAP in SCI model rats, particularly RafI, TMS+BMSCs and TMS+RafI. NGF and BDNF are neurotrophic growth factors that are critical for the development and survival of neurons (55). The results of the present study 
demonstrated that all therapies, excluding RafI monotherapy, significantly upregulated NGF and BDNF expression levels in SCI model rats compared with untreated SCI model rats. Moreover, the combination of TMS+BMSCs or TMS+RafI displayed an enhanced ability to upregulate NGF and BDNF expression levels compared with the corresponding monotherapy groups. The results suggested that combination therapies might promote neuronal growth, development and survival, and inhibit SCI-induced astroglial activation more effectively compared with monotherapies.

Emerging evidence has indicated that Raf/MEK/ERK signaling might promote the neural cell apoptosis (56). In a previous study, p-Raf/Raf, p-MEK/MEK and p-ERK/ERK expression levels were upregulated in SCI model rats, indicating that Raf/MEK/ERK signaling is involved in the development of SCI (29). C-Raf overexpression displayed an inhibitory effect on the development and maturation of spinal cord neurons $(28,29)$. In the present study, treatment with TMS, BMSCs or RafI alone inhibited SCI-induced activation of the Raf/MEK/ERK signaling pathway. Furthermore, the combined application of TMS+BMSCs or TMS+RafI displayed a significantly enhanced potential to inhibit SCI-induced activation of Raf/MEK/ERK signaling compared with the corresponding monotherapy groups. Due to the pivotal role of Raf/MEK/ERK signaling in SCI, it was hypothesized that combination therapy might display an improved therapeutic effect on SCI by further suppressing Raf/MEK/ERK signaling.

In conclusion, the results of the present study demonstrated that the therapeutic effect of TMS combined with BMSC transplantation or RafI on SCI recovery in rats was more efficient compared with monotherapy; therefore, combination therapy may serve as a novel therapeutic strategy for SCI.

\section{Acknowledgements}

Not applicable.

\section{Funding}

No funding was received.

\section{Availability of data and materials}

The datasets used and/or analyzed during the current study are available from the corresponding author on reasonable request.

\section{Authors' contributions}

LZ conceptualized and designed the study. SF performed the histological examination of the spinal cord and immunofluorescence staining and revised the manuscript. SW performed western blotting. SF and SW established the animal model of SCI and performed all treatments. SS and HS analyzed the data. HS drafted the manuscript. LZ and SF confirm the authenticity of all the raw data. All authors read and approved the final manuscript.

\section{Ethics approval and consent to participate}

All animal experiments were performed according to Guide for the Care and Use of Laboratory Animals 8th edition, and approved by the ethics committee of Shengjing Hospital of China Medical University (approval no. 2020PS697K).

\section{Patient consent for publication}

Not applicable.

\section{Competing interests}

The authors declare that they have no competing interests.

\section{References}

1. Kim YH, Ha KY and Kim SI: Spinal cord injury and related clinical trials. Clin Orthop Surg 9: 1-9, 2017.

2. Wang YT, Lu XM, Chen KT, Shu YH and Qiu CH: Immunotherapy strategies for spinal cord injury. Curr Pharm Biotechnol 16: 492-505, 2015.

3. Manchikanti L, Singh V, Datta S, Cohen SP and Hirsch JA; American Society of Interventional Pain Physicians: Comprehensive review of epidemiology, scope, and impact of spinal pain. Pain Physician 12: E35-E70, 2009.

4. Kwon BK, Tetzlaff W, Grauer JN, Beiner J and Vaccaro AR: Pathophysiology and pharmacologic treatment of acute spinal cord injury. Spine J 4: 451-464, 2004.

5. Spinal Cord Injury (SCI) 2016 facts and figures at a glance. J Spinal Cord Med 39: 493-494, 2016.

6. Ditunno JF Jr: Functional outcomes in spinal cord injury (SCI): Quality care versus cost containment. J Spinal Cord Med 20: 1-7, 1997.

7. McDonald JW and Sadowsky C: Spinal-cord injury. Lancet 359: 417-425, 2002.

8. Rowland JW, Hawryluk GW, Kwon B and Fehlings MG: Current status of acute spinal cord injury pathophysiology and emerging therapies: Promise on the horizon. Neurosurg Focus 25: E2, 2008.

9. Ren Z, Chen X, Yang J, Kress BT, Tong J, Liu H, Takano T, Zhao $\mathrm{Y}$ and Nedergaard $\mathrm{M}$ : Improved axonal regeneration after spinal cord injury in mice with conditional deletion of ephrin B2 under the GFAP promoter. Neuroscience 241: 89-99, 2013.

10. Yang T, Dai Y, Chen G and Cui S: Dissecting the dual role of the glial scar and scar-forming astrocytes in spinal cord injury. Front Cell Neurosci 14: 78, 2020.

11. Benninger F, Glat MJ, Offen D and Steiner I: Glial fibrillary acidic protein as a marker of astrocytic activation in the cerebrospinal fluid of patients with amyotrophic lateral sclerosis. J Clin Neurosci 26: 75-78, 2016.

12. Okada S, Hara M, Kobayakawa K, Matsumoto $Y$ and Nakashima Y: Astrocyte reactivity and astrogliosis after spinal cord injury. Neurosci Res 126: 39-43, 2018.

13. Curtis R, Green D, Lindsay RM and Wilkin GP: Up-regulation of GAP-43 and growth of axons in rat spinal cord after compression injury. J Neurocytol 22: 51-64, 1993.

14. Keefe KM, Sheikh IS and Smith GM: Targeting neurotrophins to specific populations of neurons: NGF, BDNF, and NT-3 and their relevance for treatment of spinal cord injury. Int J Mol Sci 18: 548, 2017.

15. Barker AT, Jalinous R and Freeston IL: Non-invasive magnetic stimulation of human motor cortex. Lancet 1: 1106-1107, 1985.

16. Pashut T, Wolfus S, Friedman A, Lavidor M, Bar-Gad I, Yeshurun $\mathrm{Y}$ and Korngreen A: Mechanisms of magnetic stimulation of central nervous system neurons. PLoS Comput Biol 7: e1002022, 2011.

17. Rothwell JC: Techniques and mechanisms of action of transcranial stimulation of the human motor cortex. J Neurosci Methods 74: 113-122, 1997.

18. Cantello R: Applications of transcranial magnetic stimulation in movement disorders. J Clin Neurophysiol 19: 272-293, 2002.

19. de Araujo AVL, Barbosa VRN, Galdino GS, Fregni F, Massetti T, Fontes SL, de Oliveira Silva D, da Silva TD, Monteiro CBM, Tonks $\mathbf{J}$ and Magalhães FH: Effects of high-frequency transcranial magnetic stimulation on functional performance in individuals with incomplete spinal cord injury: Study protocol for a randomized controlled trial. Trials 18: 522, 2017. 
20. Kumru H, Kofler M, Valls-Sole J and Vidal J: Brainstem reflex excitability after high-frequency repetitive transcranial magnetic stimulation in healthy and spinal cord injury subjects. Brain Res Bull 147: 86-91, 2019.

21. Aznar J and Sanchez JL: Embryonic stem cells: Are useful in clinic treatments? J Physiol Biochem 67: 141-144, 2011.

22. Ogawa Y, Sawamoto K, Miyata T, Miyao S, Watanabe M, Nakamura M, Bregman BS, Koike M, Uchiyama Y, Toyama Y and Okano H: Transplantation of in vitro-expanded fetal neural progenitor cells results in neurogenesis and functional recovery after spinal cord contusion injury in adult rats. J Neurosci Res 69: 925-933, 2002

23. Tsuji O, Miura K, Okada Y, Fujiyoshi K, Mukaino M, Nagoshi N, Kitamura K, Kumagai G, Nishino M, Tomisato S, et al: Therapeutic potential of appropriately evaluated safe-induced pluripotent stem cells for spinal cord injury. Proc Natl Acad Sci USA 107: 12704-12709, 2010.

24. Miao C, Lei M, Hu W, Han S and Wang Q: A brief review: The therapeutic potential of bone marrow mesenchymal stem cells in myocardial infarction. Stem Cell Res Ther 8: 242, 2017.

25. Fehlings MG and Vawda R: Cellular treatments for spinal cord injury: The time is right for clinical trials. Neurotherapeutics 8: 704-720, 2011

26. Mannoji C, Koda M, Kamiya K, Dezawa M, Hashimoto M, Furuya T, Okawa A, Takahashi $\mathrm{K}$ and Yamazaki $\mathrm{M}$ : Transplantation of human bone marrow stromal cell-derived neuroregenrative cells promotes functional recovery after spinal cord injury in mice. Acta Neurobiol Exp (Wars) 74: 479-488, 2014.

27. Roberts PJ and Der CJ: Targeting the Raf-MEK-ERK mitogen-activated protein kinase cascade for the treatment of cancer. Oncogene 26: 3291-3310, 2007.

28. Cao FJ, Zhang X, Liu T, Li XW, Malik $M$ and Feng SQ: Up-regulation of Ras/Raf/ERK1/2 signaling in the spinal cord impairs neural cell migration, neurogenesis, synapse formation, and dendritic spine development. Chin Med J (Engl) 126 $3879-3885,2013$

29. Liu T, Cao FJ, Xu DD, Xu YQ and Feng SQ: Upregulated Ras/Raf/ERK1/2 signaling pathway: A new hope in the repair of spinal cord injury. Neural Regen Res 10: 792-796, 2015.

30. Sugaya T, Kanno H, Matsuda M, Handa K, Tateda S, Murakami T, Ozawa $\mathrm{H}$ and Itoi E: B-RAF(V600E) inhibitor dabrafenib attenuates RIPK3-mediated necroptosis and promotes functional recovery after spinal cord injury. Cells 8: 1582, 2019.

31. Woller SA, Malik JS, Aceves M and Hook MA: Morphine self-administration following spinal cord injury. J Neurotrauma 31: 1570-1583, 2014.

32. Basso DM, Beattie MS and Bresnahan JC: Graded histological and locomotor outcomes after spinal cord contusion using the NYU weight-drop device versus transection. Exp Neurol 139 244-256, 1996.

33. Sandhir R, Gregory E, He YY and Berman NE: Upregulation of inflammatory mediators in a model of chronic pain after spinal cord injury. Neurochem Res 36: 856-862, 2011.

34. National Research Council (US) Committee for the Update of the Guide for the Care and Use of Laboratory Animals: Guide for the Care and Use of Laboratory Animals, 8th edition. National Academies Press (US), Washington, DC, 2011.

35. Chen Q, Duan X, Xu M, Fan H, Dong Y, Wu H, Zhang M, Liu Y, Nan Z, Deng S and Liu X: BMSC-EVs regulate Th17 cell differentiation in UC via H3K27me3. Mol Immunol 118: 191-200, 2020.

36. Eckert MJ and Martin MJ: Trauma: Spinal cord injury. Surg Clin North Am 97: 1031-1045, 2017.

37. Lee BB, Cripps RA, Fitzharris M and Wing PC: The global map for traumatic spinal cord injury epidemiology: Update 2011, global incidence rate. Spinal Cord 52: 110-116, 2014.

38. Awad BI, Carmody MA, Zhang X, Lin VW and Steinmetz MP. Transcranial magnetic stimulation after spinal cord injury. World Neurosurg 83: 232-235, 2015.

39. Cha HG, Ji SG and Kim MK: Effect of high-frequency repetitive transcranial magnetic stimulation on motor cortical excitability and sensory nerve conduction velocity in subacute-stage incomplete spinal cord injury patients. J Phys Ther Sci 28: 2002-2004, 2016.
40. Lin L, Lin H, Bai S, Zheng L and Zhang X: Bone marrow mesenchymal stem cells (BMSCs) improved functional recovery of spinal cord injury partly by promoting axonal regeneration. Neurochem Int 115: 80-84, 2018.

41. Wu S, Suzuki Y, Ejiri Y, Noda T, Bai H, Kitada M, Kataoka K, Ohta $\mathrm{M}$, Chou $\mathrm{H}$ and Ide $\mathrm{C}$ : Bone marrow stromal cells enhance differentiation of cocultured neurosphere cells and promote regeneration of injured spinal cord. J Neurosci Res 72: 343-351, 2003.

42. Wang F, Zhang C, Hou S and Geng X: Synergistic effects of mesenchymal stem cell transplantation and repetitive transcranial magnetic stimulation on promoting autophagy and synaptic plasticity in vascular dementia. J Gerontol A Biol Sci Med Sci 74: $1341-1350,2019$

43. Anderson KD, Abdul M and Steward O: Quantitative assessment of deficits and recovery of forelimb motor function after cervical spinal cord injury in mice. Exp Neurol 190: 184-191, 2004.

44. Basso DM, Beattie MS and Bresnahan JC: A sensitive and reliable locomotor rating scale for open field testing in rats. J Neurotrauma 12: 1-21, 1995.

45. Poirrier AL, Nyssen Y, Scholtes F, Multon S, Rinkin C, Weber G, Bouhy D, Brook G, Franzen R and Schoenen J: Repetitive transcranial magnetic stimulation improves open field locomotor recovery after low but not high thoracic spinal cord compression-injury in adult rats. J Neurosci Res 75: 253-261, 2004.

46. Ning GZ, Song WY, Xu H, Zhu RS, Wu QL, Wu Y, Zhu SB, Li JQ, Wang M, Qu ZG and Feng SQ: Bone marrow mesenchymal stem cells stimulated with low-intensity pulsed ultrasound: Better choice of transplantation treatment for spinal cord injury: Treatment for SCI by LIPUS-BMSCs transplantation. CNS Neurosci Ther 25: 496-508, 2019.

47. Elmore S: Apoptosis: A review of programmed cell death. Toxicol Pathol 35: 495-516, 2007.

48. Mazarakis ND, Edwards AD and Mehmet $\mathrm{H}$ : Apoptosis in neural development and disease. Arch Dis Child Fetal Neonatal Ed 77: F165-F170, 1997.

49. Kim DH, Vaccaro AR, Henderson FC and Benzel EC: Molecular biology of cervical myelopathy and spinal cord injury: Role of oligodendrocyte apoptosis. Spine J 3: 510-519, 2003.

50. Lan WB, Lin JH, Chen XW, Wu CY, Zhong GX, Zhang LQ, Lin WP, Liu WN, Li X and Lin JL: Overexpressing neuroglobin improves functional recovery by inhibiting neuronal apoptosis after spinal cord injury. Brain Res 1562: 100-108, 2014.

51. Zhang H, Wu F, Kong X, Yang J, Chen H, Deng L, Cheng Y, Ye L, Zhu S, Zhang X, et al: Nerve growth factor improves functional recovery by inhibiting endoplasmic reticulum stress-induced neuronal apoptosis in rats with spinal cord injury. J Transl Med 12: 130, 2014.

52. Rong W, Wang J, Liu X, Jiang L, Wei F, Hu X, Han X and Liu Z: Naringin treatment improves functional recovery by increasing BDNF and VEGF expression, inhibiting neuronal apoptosis after spinal cord injury. Neurochem Res 37: 1615-1623, 2012.

53. Madsen JR, MacDonald P, Irwin N, Goldberg DE, Yao GL, Meiri KF, Rimm IJ, Stieg PE and Benowitz LI: Tacrolimus (FK506) increases neuronal expression of GAP-43 and improves functional recovery after spinal cord injury in rats. Exp Neurol 154: 673-683, 1998.

54. Brenner M, Kisseberth WC, Su Y, Besnard F and Messing A GFAP promoter directs astrocyte-specific expression in transgenic mice. J Neurosci 14: 1030-1037, 1994.

55. Blais M, Levesque $P$, Bellenfant $S$ and Berthod F: Nerve growth factor, brain-derived neurotrophic factor, neurotrophin-3 and glial-derived neurotrophic factor enhance angiogenesis in a tissue-engineered in vitro model. Tissue Eng Part A 19: 1655-1664, 2013.

56. Feng D, Wang B, Ma Y, Shi W, Tao K, Zeng W, Cai Q, Zhang Z and Qin H: The Ras/Raf/erk pathway mediates the subarachnoid hemorrhage-induced apoptosis of hippocampal neurons through phosphorylation of p53. Mol Neurobiol 53: 5737-5748, 2016.

This work is licensed under a Creative Commons Attribution-NonCommercial-NoDerivatives 4.0 International (CC BY-NC-ND 4.0) License. 